\title{
Analysis on the Driving Effect of Scientific and Technological Innovation on the Coordinated Development of Regional Economy
}

\author{
Jialiang Chen \\ University of Pennsylvania, Philadelphia, PA, USA
}

\begin{abstract}
This paper studies the driving effect of scientific and technological innovation on regional economic development. From the perspective of scientific and technological innovation, it can promote the transformation and upgrading of industrial structure, as well as expand the consumer demand and investment demand. The countermeasures of driving regional economic development by scientific and technological innovation were put forward: to advocate independent innovation of regional enterprises; to attach importance to the development of new culture; to constantly optimize the Top-level design, and to build the coordinated development model of regional economy with systematic thinking; to improve government policy support and guidance.
\end{abstract}

Keywords: Scientific and Technological Innovation; Driving Effect; Regional Economy.

\section{The Influence and Enlightenment of World Scientific and Technological Innovation on Social and Economic Development}

From the impact of scientific and technological innovation on social and economic development in the world, it can be found that scientific and technological innovation can play an important driving role. The economic take-off of modern countries was accompanied by scientific and technological innovation. From the first scientific and technological revolution in the 18th century, to the steam engine in Britain and to the fifth scientific and technological revolution led by the United States in the 1980s and 1990s, the development of information industrialization and Internet economy, human society has experienced five "long waves" of science and technology. Each has established the world leadership of the country leading the wave and brought "nuclear fusion" influence to the world economic and social development.

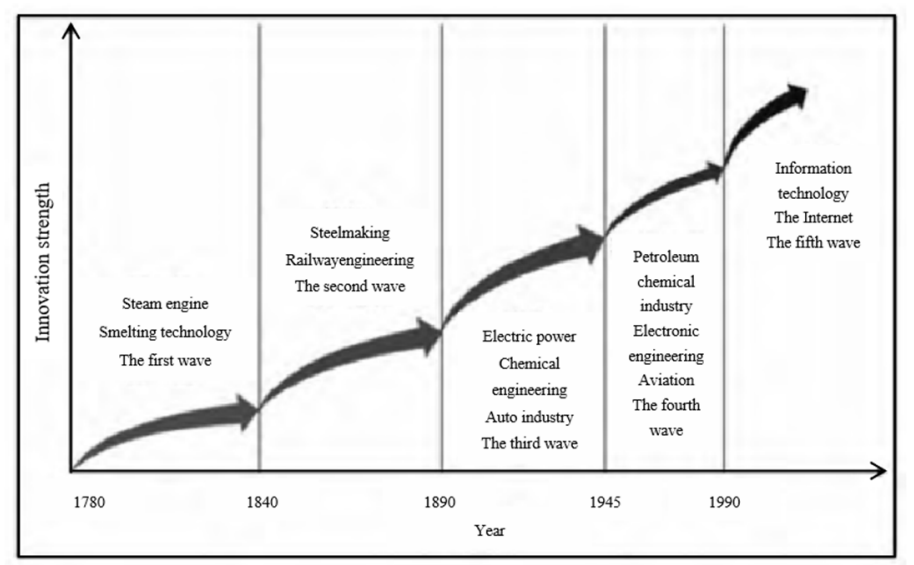

Figure 1. Five waves of scientific and technological revolution

In the 1980s, Apple and Microsoft became the promoters of computer development, driving the development of social information industry. In the future, with the development of new technologies such as big data and Internet of Things, the world will usher in the era of cloud + big data and Internet of Things/intelligent hardware. In the face of the historic convergence of scientific and technological revolution and industrial transformation in the world, and increasingly fierce competition to master 
the greatest advantage of the future, the traditional growth model driven by factor input is no longer sustainable, and the task of building an innovative country remains a long and arduous one. In this context, China has reached a new stage in which it must rely more on scientific and technological innovation to guide and support economic development and social progress.[1]

\section{Theories Related to Regional Economic Development Driven by Scientific and Technological Innovation}

\subsection{Theory of Regional Scientific and Technological Innovation}

Theory of regional scientific and technological innovation mainly includes regional scientific and technological innovation environment, regional scientific and technological innovation concept as well as regional scientific and technological innovation structure and capability. Among them, the regional scientific and technological innovation capability not only contains the actual capability and potential capability, but also involves the analysis of the impact of regional scientific and technological innovation on regional economic development. Its research focuses on the contribution of regional scientific and technological innovation to the promotion of regional competitiveness and economic growth. According to the theory, scientific and technological innovation and progress become the important driving force for the sustainable development of regional economy, and the differences in the speed of scientific and technological progress and the efficiency of scientific and technological innovation are also the main causes for the disparities in regional economic development.[2]

\subsection{Theory of Regional Economic Development}

The theory of regional economic development includes balanced development theory and unbalanced development theory. The former claims that regional economic development will eventually reach a balanced state, while the latter holds that in the absence of external factors, it is difficult for regional economic development to reach equilibrium, and the differences will continue to increase. In addition, the theory of regional economic development difference supports that labor force, capital and technology are the decisive factors of regional economic development. Under the condition of diminishing marginal returns and free flow of factors of production, if the technological level and economic preference are the same, the growth rate of backward regions is faster than that of developed economies, and then the regional economic development differences tend to converge. On the contrary, if there is "Matthew effect", it will make the differences in regional economic development become larger and larger.

\section{Driving Effect of Scientific and Technological Innovation on Regional Economic Development}

\subsection{Scientific and Technological Innovation can Expand Consumer Demand and Investment Demand}

At present, under the modern market economy, the important factors driving economic growth are consumption, investment as well as import and export, whose demand changes affect China's GDP growth. In order to promote economic development reasonably, attention must be paid to adjusting the proportion of consumption and investment, and in the consumption structure, scientific and technological innovation should be applied to boosting consumption. For example, the mobile phone industry should constantly optimize products through scientific and technological innovation, and introduce $4 \mathrm{G}$ and $5 \mathrm{G}$ mobile phones to change consumer demand. Based on the increasing demand, it drives the change of industrial structure. When investors realize the growth potential of the industry, they will increase the proportion of investment accordingly. 


\subsection{Scientific and Technological Innovation can Promote Industrial Structure Transformation and Upgrading}

China's industrial development is faced with an important issue of unreasonable and incongruous structure. From the aspect of economic proportion, the proportion of tertiary industry is very low, never more than 50\%. By contrast, developed countries have built a road of economic development based on high and new technology, and their tertiary industry accounts for about $75 \%$ of the national industrial structure. In recent years, the rapid development of communication industry and information industry has driven the optimization and adjustment of their industrial structure and contributed to the rapid growth of GDP. It can be said that it is the scientific and technological innovation that drives their industrial structure adjustment and maintains rapid economic growth. From the perspective of China's industrial structure optimization, on the premise of not changing the first structure, the industrial structure optimization should be deepened, and the innovative process production mode should be implemented, so as to promote the increase in the proportion of the tertiary industry. With cloud computing technology as an example, relying on cloud computing to complete industrial transformation and upgrading, and the specific modes are as follows: (1) Integration mode should be conducted. Cloud computing should be organically integrated into relevant fields, such as traditional production, so that it can be organically integrated with the information industry to form an industrial structure with development value. (2) Conversion mode should be carried out. With the help of High-tech technology, it will help optimize the development of traditional industries, and then promote the optimization, adjustment and upgrading of industrial structure, such as smart agriculture.[3]

\section{Scheme of Scientific and Technological Innovation to Drive the Coordinated Development of Regional Economy}

\subsection{Vigorously Advocating the Independent Innovation of Regional Enterprises}

From the perspective of the development power source of regional economy, enterprise is an important "engine", so in order to give full play to the role of scientific and technological innovation in driving regional economic development, the independent innovation of regional enterprises should be vigorously advocated. In the new era, only by actively changing the mode of economic development and grasping the initiative in market competition can the sustainable and stable growth of regional economy be maintained. Effectively transforming the economic development model requires to highlight the function of scientific and technological innovation, intensify independent innovation and create new economic growth points. For enterprises, having strong independent innovation capacity is of great significance to improve their market competitiveness, drive industry innovation and promote the development of innovative country. Enterprise innovation should be well controlled in the following aspects: (1) The innovation awareness of staff should be enhanced. The continuous improvement of independent innovation of enterprises requires the participation of all employees, giving play to their wisdom, as well as concentrating innovation and creativity, so as to achieve breakthroughs. (2) The investment in innovation should be increased. According to the actual needs of project research and development, corresponding support should be given, especially capital and technology, to facilitate scientific and technological innovation. (3) Incentives should be fully applied.

\subsection{Attaching Importance to the Development of New Culture}

The sustainable development of economy needs not only the drive of scientific and technological innovation, but also the support and guarantee of culture. By introducing and cultivating new culture, the sustainable development of regional economy can be promoted. In recent years, China's scientific and technological innovation has achieved major breakthroughs, obtaining good results in various 
key projects and projects frequently, such as the supercomputers and rice hybrid. The achievements of scientific and technological innovation provide an important guarantee and support for the sustained growth of regional economy. In terms of achievements of scientific and technological innovation, they carry profound cultural significance and help the sustainable development of various industries. For the cultivation of innovation culture, it is necessary to foster the habit of innovation, enhance the innovation consciousness of individuals and enterprises, and make innovation a common behavior. Besides, the innovation culture should be actively publicized to enhance people's sense of identity and pride in it, so that innovation can be penetrated into people's mind.

\subsection{Constantly Optimizing the Top-level Design and Building a Coordinated Development Model of Regional Economy with Systematic Thinking}

At the national level, it is suggested to establish a multi-dimensional and multi-scale assessment system for regional or government development and innovation, and guide governments at all levels to lay emphasis on scientific and technological innovation as a driving force for promoting regional economic development. From the dimensions of development, coordination and sustainability, it is suggested to establish a diversified government evaluation system integrating scientific and technological innovation index and economic indicators, attach importance to scientific planning of resources and environment development, quantify the current GDP assessment index, and give prominence to the measurement of scientific and technological innovation index. Therefore, relevant departments should strengthen the research on the index system of national scientific and technological economic innovation, and intensify the research on the scientific and objective influence of the index system of scientific and technological innovation on regional economic development.

\subsection{Improving the Government Policy Support and Guidance}

At present, the eastern China has the most abundant innovation resources, with stronger innovation capacity as well as obviously higher economic development level. Meanwhile, the scientific and technological innovation in the western China is also improving continuously and has a strong driving effect on economic development. In order to strengthen the driving force of scientific and technological innovation on regional economic growth in the long term, it is necessary to roll out corresponding policies according to the status of scientific and technological innovation and economic development in each region, strengthen the support of financial funds, reinforce the correct guidance of scientific and technological innovation, give full play to its agglomeration effect and demonstration effect, fully drive the sustainable development of all industries, and take scientific and technological innovation as the most critical factor to promote the sustainable economic growth of each region.[4]

\subsubsection{Further Increasing the Investment in Science and Technology in Western China to Realize its Leapfrog Development}

The development of western China needs to move from the large-scale development stage to the leapfrog development stage. Accordingly, it is suggested that the optimization planning of scientific and technological resources and R\&D investment in western China should be systematically established at the national and regional levels. The "Outline of Western Leapfrog Development Plan" or "Western Leapfrog Collaborative Innovation Action Plan" should be issued at the national level to better coordinate and promote balanced regional development.

\subsubsection{Constructing Innovative Environment to Support the Innovation of Characteristic High- tech Industry in Western China}

The western region shows strong development advantages in pharmaceutical manufacturing, aviation, spacecraft and equipment manufacturing, and its research and development strength and potential are comparable to that of the central and eastern regions. It is suggested to continue to strengthen the integration of the advantageous industry and scientific and technological innovation to 
build a sustainable development mode of regional economic competitiveness with scientific and systematic thinking, and make the technology service of characteristic industry become the greatest advantage of service brand in each region.

\subsubsection{Establishing the Linkage Mechanism and Platform for Regional Characteristic Industries}

All regions should actively carry out open cooperation in scientific and technological innovation, optimize their own scientific and technological strategic development by taking advantage of resources and intelligence advantages of institutions outside the region, to enhance their scientific and technological innovation capabilities. They should establish a linkage mechanism and platform for regional characteristic industries, strengthen inter-regional cooperation in science and technology industries, and build the characteristic industrial strategy consulting think tank of government, industry, university and research and relevant development environment.

\section{Conclusion}

Sustainable coordinated development of regional economy is a systematic management project. It is suggested to establish a path at the national level to guide each region to attach importance to the basic conditions of scientific and technological innovation in industrial development, stress the layout and development of disciplines, and further carry out Top-level design for the central and western regions. From the dimensions of development, coordination and sustainability, it is suggested to guide the dislocated and complementary development of the eastern, central and western regions, and fully reflect the characteristics of functional zones.

\section{References}

[1] Shaopeng Wang, Xinru Miao, Zenglei Xi. Scientific and Technological Innovation, Spatial Spillover and Regional Economic Development in Colleges and Universities[J]. Technology Economics,2021, 40 (4): $49-57$.

[2] Jiawen Li, Bin Guo, Research on Coupling Coordination Degree and Spatio-temporal Differentiation of Scientific and Technological Innovation in Colleges and Universities and Regional Economy Development [J]. Technology Economics,2020, 39(4):112-119.

[3] Lin Li, Zhihua Liu, Yuwen Jiang. Research on Regional Scientific and Technological Synergy Innovation of Central Hunan Economic Circle [J]. Journal of Hunan University (Social Sciences),2014, 28(2):47-52.

[4] Deyu Chen, Yonggang Tang, Shaohe Zhang. Empirical Analysis of Industrial Structure Transformation and Upgrading, Financial Science and Technology Innovation and Regional Economic Development[J]. Science and Technology Management Research,2018, 38(15):105-110. 